\title{
Inverse Method for Determining Material Parameters of Piezoceramics and Passive Materials
}

\author{
Manuel Weiß ${ }^{1}$, Jürgen $I l g^{1}$, Stefan J. Rupitsch ${ }^{1}$, Reinhard Lerch ${ }^{1}$ \\ 1 Friedrich-Alexander University of Erlangen-Nürnberg, Chair of Sensor Technology, \\ Paul-Gordan-Str. 3/5, 91052 Erlangen, Germany \\ manuel.weiss@fau.de
}

\begin{abstract}
We present an Inverse Method for determining material parameters of piezoceramics as well as passive materials (e.g., metals, glasses, plastics, composites). The Inverse Method is based on the minimization of the deviation between measurements and simulation results by an optimization algorithm. In doing so, material parameters are identified via an iterative adjustment of the simulation parameters. Contrary to common characterization methods, the Inverse Method is qualified to identify all material parameters of piezoceramics (i.e., tensors of elasticity, permittivity and piezoelectric coupling) through measured electrical impedances of only two test samples. Furthermore, the Inverse Method is applied for determining frequency dependent material parameters (e.g., tensor of elasticity, damping factor) of passive materials by forced vibration testing. We show results for a characterized piezoelectric material and a passive material.
\end{abstract}

Key words: Inverse Method, piezoceramics, viscoelasticity, and characterization

\section{Introduction}

The availability of precise material parameters is essential for FEM-based (finite element method) numerical simulations. Consequently, the determination of material parameters is of major importance. Previous publications have shown suitable procedures to characterize material parameters of piezoceramics $[1,2,5,7]$ and passive materials $[4,8]$. In this contribution two adapted approaches are illustrated which provide material parameters of piezoceramics and passive materials by means of the Inverse Method. Section 2 addresses the fundamentals of the Inverse Method. Section 3 deals with the basics of piezoceramics, the used measurement setup and results of the characterized piezoelectric material SONOX® P5.

The determination of frequency dependent material parameters of passive materials by an adapted Inverse Method, developed as part of a doctoral thesis [6], is illustrated in section 4. At this, a phenomenological approach is introduced, which is applied to determine the frequency dependent behavior. Finally, the frequency dependence of PVC is shown.

\section{Fundamentals of the Inverse Method}

The Inverse Method is a suitable tool for solving ill-posed problems with a known effect (i.e., measurement results, boundary conditions and excitation) but unknown cause (i.e., material parameters). Unlike direct problems, which mostly enable a solution by calculation, the Inverse Method finds the solution stepwise by means of appropriate regularization methods.

Figure 1 shows a flowchart of the Inverse Method. In order to identify all desired material parameters, measurements and simulations of selected physical quantities of the specimen are required. We conduct numerical simulations in the finite element tool CFS++ (Coupled Field Simulation). An initial material parameter set (4) is required to run the first simulations (i.e., manufacturer data). After each simulation, the deviation between measurements and simulations is determined (6). In addition, adjusted material parameters are calculated through a regularization algorithm (e.g., Levenberg-Marquardt, Gauß-Newton).

Afterwards, the next simulation with an adapted parameter set is started. This procedure continues until an abort criteria (8) is fulfilled. That means the deviation between measurement and simulation results become very small and all parameters converge towards a certain value. Sensitivity studies ensure that the Inverse Method finds the global minimum. 


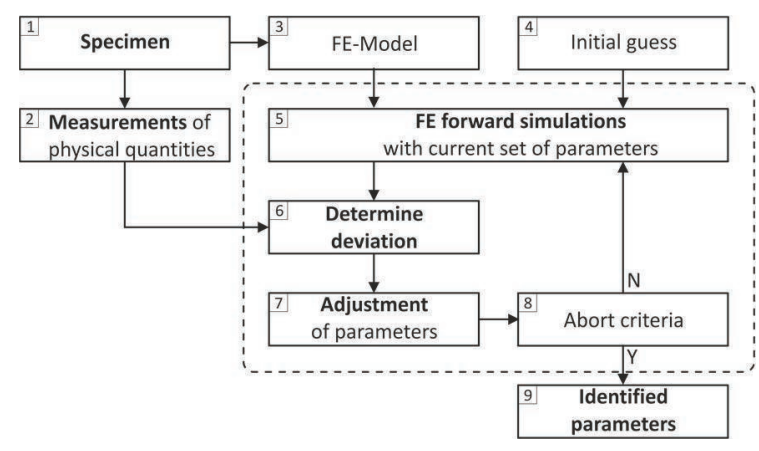

Fig. 1: Flowchart of the Inverse Method.

\section{Identification of material parameters for piezoceramics}

The piezoelectric characteristic of materials is defined by the piezoelectric equations

$\vec{D}=\boldsymbol{e} \vec{S}+\boldsymbol{\varepsilon}^{S} \vec{E}$

$\vec{T}=c^{E} \vec{S}-e^{\mathrm{t}} \vec{E}$,

which describe the relationship between mechanical and electrical behavior $(\vec{D}$ : dielectric displacement, $\vec{E}$ : electric field, $\vec{S}$ : mechanical strain, $\vec{T}$ : mechanical stress). An elasticity tensor, a permittivity tensor and a tensor of piezoelectric coupling is required. For class $6 \mathrm{~mm}$ materials, these tensors are defined as [3]

$\boldsymbol{c}^{E}=\left[\begin{array}{cccccc}c_{11}^{E} & c_{12}^{E} & c_{13}^{E} & 0 & 0 & 0 \\ & c_{11}^{E} & c_{13}^{E} & 0 & 0 & 0 \\ & & c_{33}^{E} & 0 & 0 & 0 \\ S & & & c_{44}^{E} & 0 & 0 \\ & Y & & & c_{44}^{E} & 0 \\ & & M & & & \frac{c_{11}^{E}-c_{12}^{E}}{2}\end{array}\right]$

$\boldsymbol{\varepsilon}^{S}=\left[\begin{array}{ccc}\varepsilon_{11}^{S} & 0 & 0 \\ 0 & \varepsilon_{11}^{S} & 0 \\ 0 & 0 & \varepsilon_{33}^{S}\end{array}\right]$

$\boldsymbol{e}=\left[\begin{array}{cccccc}0 & 0 & 0 & 0 & e_{15} & 0 \\ 0 & 0 & 0 & e_{15} & 0 & 0 \\ e_{31} & e_{31} & e_{33} & 0 & 0 & 0\end{array}\right]$

The tensors contain ten independent material parameters. Additionally, a loss factor $\alpha$ is required to consider damping. To achieve reliable finite element based simulation results, all eleven parameters are required.

\subsection{Measurements}

Contrary to common characterization methods [3], the Inverse Method is qualified to identify all material parameters of piezoceramics through measured electrical impedances of only two test samples. Those specimens only differ in their polarization direction: a thickness mode sample (T1), polarized in thickness direction, and a thickness shear mode sample (T2), polarized in width direction. The impedance around three characteristic resonance frequencies of sample T1 (T1-L, T1-W, T1-T) and one resonance frequency of sample $\mathrm{T} 2$ is measured by an impedance analyzer (HP 4192A). Thereby, two spring pins connect the samples (see Fig. 2). The measurements are realized inside an environmental chamber to additionally determine temperature dependence of the material parameters [5].

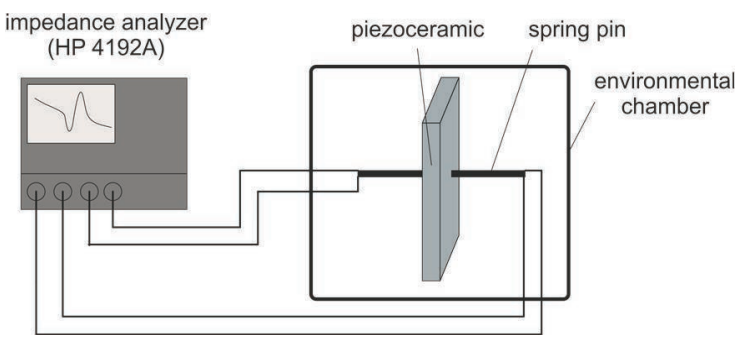

Fig. 2: Impedance measurement setup.

\subsection{Simulations}

Similar to the measurements, we simulate electrical impedances around all four resonance frequencies. In doing so, three different simulation models are utilized: a 3D model for transverse length modes T1-L and T1-W, a 2D model for thickness extensional mode T1-T and a $2 \mathrm{D}$ model with a rotated polarization direction for the thickness shear mode T2 [1]. Note that the simplification of the $3 \mathrm{D}$ models to $2 \mathrm{D}$ models is more efficient due to the many elements required for higher frequency simulations.

\subsection{Results}

The frequency resolved electrical impedance of the simulations with the identified parameter set coincides well with the measurements (see Fig. 3 ). Table 1 shows average values and deviations of identified parameters for the piezoelectric material Sonox® P5 (CeramTec) at $20^{\circ} \mathrm{C}$. For this, five samples of type $\mathrm{T} 1$ and five samples of type T2 were measured at identical conditions. Thus, 25 Inverse Methods were executed to generate statistical information. 
Fig. 3: Electrical impedance at resonance frequencies of thickness (T1) and shear (T2) mode sample. Measurement (solid line), simulation with initial guess parameter (dotted), simulation with identified parameter set (dashed).
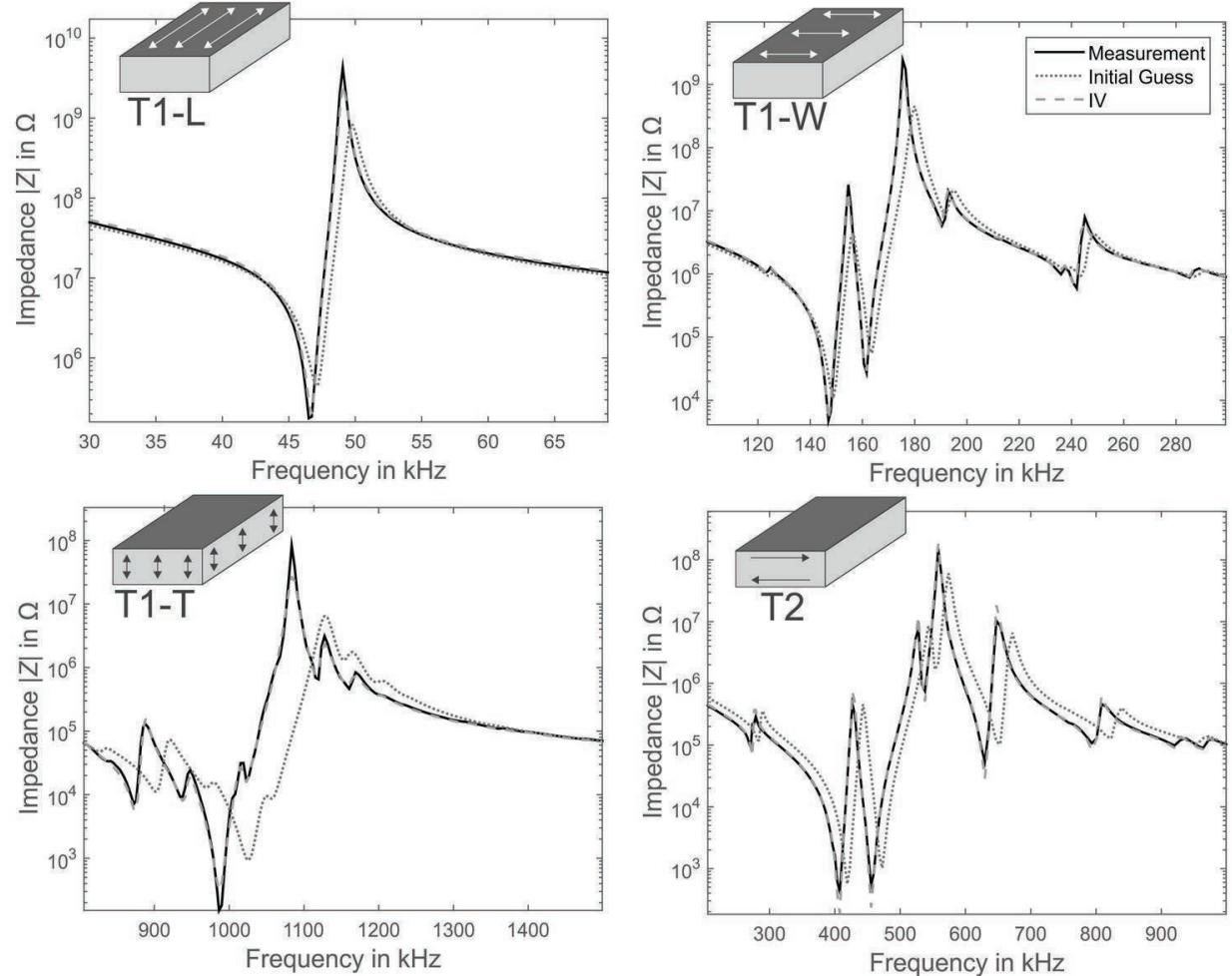

Tab. 1: Identified parameters of CeramTec Sonox® P5 at $20^{\circ} \mathrm{C} ; c_{x y}^{E}$ in $\mathrm{N} / \mathrm{m}^{2} ; \varepsilon_{x y}^{S}$ in $\mathrm{F} / \mathrm{m} ; e_{x y}$ in $\mathrm{C} / \mathrm{m}^{2}$. Average values and standard deviations on basis of 25 identification results.

\begin{tabular}{|c|c|c|c|c|c|c|c|c|c|c|c|}
\hline & \multicolumn{10}{|c|}{ Parameters } \\
\hline & $c_{11}^{E}$ & $c_{12}^{E}$ & $c_{13}^{E}$ & $c_{33}^{E}$ & $c_{44}^{E}$ & $\varepsilon_{11}^{S}$ & $\varepsilon_{33}^{S}$ & $e_{31}$ & $e_{33}$ & $e_{15}$ & $\alpha$ \\
\hline Average & $122.4 \cdot 10^{9}$ & $76.2 \cdot 10^{9}$ & $76.5 \cdot 10^{9}$ & $110.9 \cdot 10^{9}$ & $19.1 \cdot 10^{9}$ & $7.8 \cdot 10^{-9}$ & $6.4 \cdot 10^{-9}$ & -5.5 & 15.1 & 12.2 & 0.012 \\
\hline $\begin{array}{c}\text { Standard } \\
\text { deviation } \\
\text { in \% }\end{array}$ & 0.3 & 0.4 & 0.4 & 0.5 & 0.4 & 0.5 & 2.5 & 1.4 & 1.3 & 0.4 & 2.3 \\
\hline
\end{tabular}

\section{Identification of frequency dependent parameters of passive materials}

Constant material parameters of passive materials often lead to imprecise numerical simulation results. In contrast, frequency dependent material parameters can be applied to simulate viscoelastic behavior, especially important for plastics. To take frequency dependent behavior into account, we use a phenomenological approach [6]. Despite several advantages, the implementation of fractional derivatives [9] is not yet expedient for our applications due to a higher complexity and the need of an additional measurement at high frequencies [6].

The mechanical behavior of a sample is described by the Hooke's law, which contains the elasticity tensor. For isotropic materials, the elasticity tensor

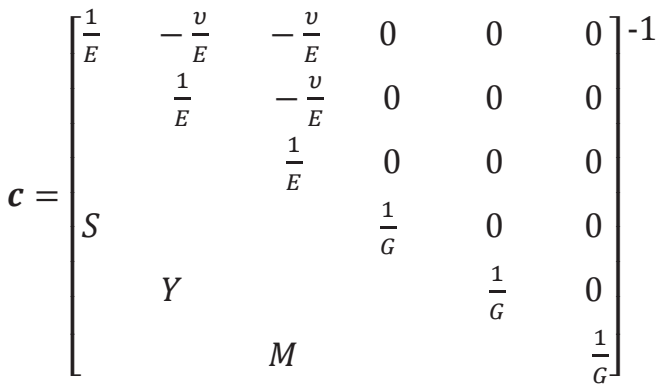

$G=\frac{E}{2(1+v)}$

possesses two independent material parameters: the elasticity modulus $E$ and the 
Poisson's ratio $v$. Furthermore, a damping factor $\xi$ is defined.

The phenomenological approach to consider viscoelasticity consists of a static, a linear and a logarithmic term (see Eq. 8). The Poisson's ratio is assumed as constant. The frequency dependence of the damping factor results from the elasticity modulus through the KramersKronig relationship (see Eq. 9-12) [6, 9, 10]. The precondition for this are causal functions of elasticity modulus and damping factor. Causal means that the response of a system is only influenced by previous or current events. Ilg [6] shows a detailed consideration on causality of the applied approach. Parameter $b$ is added to Eq. 12 through the underlying approximation (Eq. 9) [6].

$$
\begin{aligned}
& E_{\mathrm{Re}}(f)=E_{0}\left[1+a_{1} \cdot f+a_{2} \cdot \log \left(\frac{f+1 \mathrm{~Hz}}{\mathrm{~Hz}}\right)\right] \\
& E_{\mathrm{Im}}(\omega) \approx \frac{\pi}{2} \omega \frac{\mathrm{d}\left[E_{\mathrm{Re}}(\omega)\right]}{\mathrm{d} \omega} \\
& E_{\operatorname{Im}}(f) \approx \frac{\pi}{2} E_{0}\left[a_{1} \cdot f+\frac{a_{2}}{\ln 10} \frac{f}{f+1 \mathrm{~Hz}}\right] \\
& \xi(\mathrm{f})=\frac{E_{\mathrm{Im}}}{2 \cdot E_{\mathrm{Re}}} \\
& \xi(\mathrm{f}) \approx b \frac{\pi\left[a_{1} \cdot f+\frac{a_{2}}{\ln 10} \frac{f}{f+1 \mathrm{~Hz}}\right]}{4\left[1+a_{1} \cdot f+a_{2} \cdot \log \left(\frac{f+1 \mathrm{~Hz}}{\mathrm{~Hz}}\right)\right]}
\end{aligned}
$$

Note that this approach is only partially applicable for transient simulations because causality is just ensured for frequencies surrounding the measurement frequency range (5 Hz - $10 \mathrm{kHz}$ ) [6].

\subsection{Measurements}

The Vibration Transmission Analyzer (VTA) determines a frequency dependent transfer function

$|H|=\left|\frac{v_{\mathrm{M}}}{v_{\mathrm{R}}}\right|$

of an oscillating specimen (see Fig. 4). The sample is stimulated harmonically by an electromechanical shaker in a frequency range of $5 \mathrm{~Hz}$ to $10 \mathrm{kHz}$. In doing so, the velocity near clamping and free running end of the specimen is measured by means of laser Doppler vibrometers $[4,6]$.

Additionally, the influence of temperature on the frequency dependent material parameters is identified via placing specimen inside an environmental chamber $\left(\vartheta=-40^{\circ} \mathrm{C}\right.$ to $\left.+140^{\circ} \mathrm{C}\right)$.

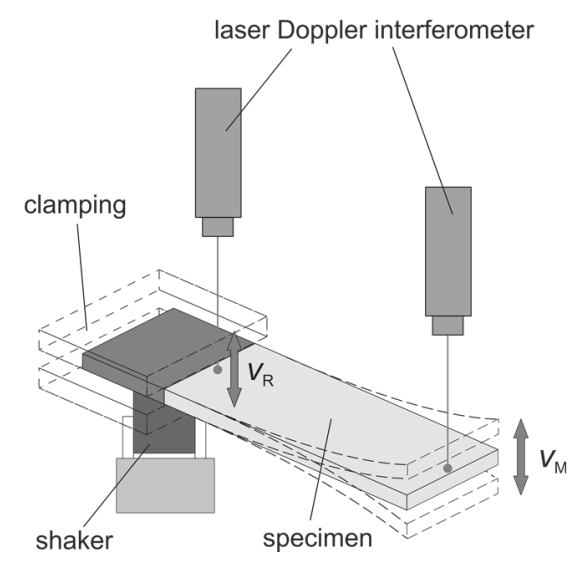

Fig. 4: Principle to acquire velocity transfer function by the Vibration Transmission Analyzer (VTA).

\subsection{Simulations}

Similar to the characterization of piezoceramics by the Inverse Method, numerical simulations are required, which are also realized with CFS++. However, we utilize anisotropic 3D pFEM (higher order hierarchic finite elements) ansatz functions to simulate thin specimens in an efficient way [6].

\subsection{Enhanced Inverse Method}

The material characterization of passive materials is performed in three substeps. This enables the Inverse Method to identify precise parameter values with lower requirements for the initial guess parameters. Firstly, an approximated elasticity modulus is found by eigenfrequency analysis and extrapolation for the first five resonance frequencies. Subsequently, the elasticity modulus and damping is determined around the first five resonance frequencies by means of harmonic simulations. Finally, the above-mentioned frequency dependent elasticity modulus, damping factor (see Eq. 8 and 12) and a constant Poisson's ratio are identified over the whole frequency range $[4,6]$.

\subsection{Results}

Figure 5 illustrates the frequency dependent material parameters elasticity modulus $E(f)$ and damping factor $\xi(f)$ of PVC. The frequency range is limited to $4 \mathrm{kHz}$ since multidimensional clamping vibrations can influence higher frequency measurements (dependent on material). The simulation result with identified parameters is improved significantly as compared to simulations with static manufacturer data. At this point, it should be noted that frequency dependent parameters can be applied to predict the real behavior also for higher frequencies satisfactorily. 

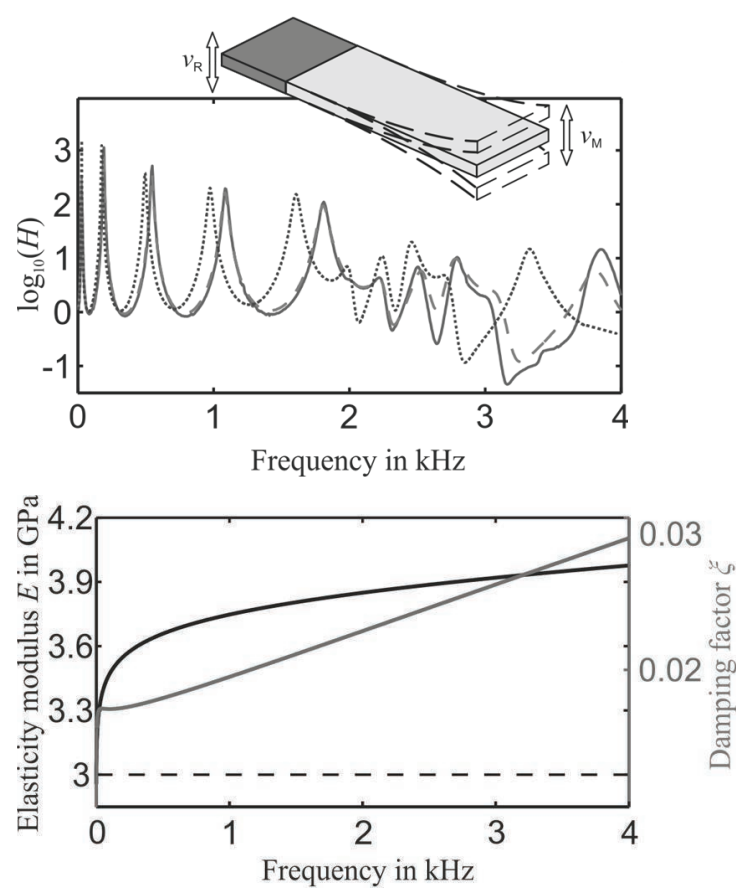

Fig. 5: Results of characterized PVC specimen. Top: Transfer functions (- measurement, -- simulation result with identified parameters, $\cdots$ simulation with manufacturer data). Bottom: Frequency dependent elasticity modulus $E$ and damping factor $\xi$ versus frequency (-- manufacturer data) [6].

\subsection{Error estimation}

Three different Inverse Methods are performed to estimate the maximal error caused by measurement conditions. Table 2 shows the specifications of the utilized FE models. The estimated worst-case parameter deviations yield amplitude changes and resonance shifts to higher or lower frequencies. Figure 6 illustrates the tolerance range estimation of an exemplary PTFE sample $(I=149.99 \mathrm{~mm}$, $b=39.99 \mathrm{~mm}, h=4.03 \mathrm{~mm}$ ).

Tab. 2: Specification of error estimation [6].

\begin{tabular}{|c|c|c|c|}
\hline & Case 1 & Case 2 & Case3 \\
\hline Length & $I$ & $I+0.2 \mathrm{~mm}$ & $I-0.2 \mathrm{~mm}$ \\
\hline Width & $b$ & $b-0.2 \mathrm{~mm}$ & $b+0.2 \mathrm{~mm}$ \\
\hline Thickness & $h$ & $h-0.05 \mathrm{~mm}$ & $h+0.05 \mathrm{~mm}$ \\
\hline Clamping length & $k$ & $k-0.1 \mathrm{~mm}$ & $k+0.1 \mathrm{~mm}$ \\
\hline Meas. point $(\mathrm{x})$ & $m_{x}$ & $m_{x}-1 \mathrm{~mm}$ & $m_{x}+1 \mathrm{~mm}$ \\
\hline Meas. point $(\mathrm{y})$ & $m_{y}$ & $m_{y}-1 \mathrm{~mm}$ & $m_{y}+1 \mathrm{~mm}$ \\
\hline Mass & $52.65 \mathrm{~g}$ & $52.66 \mathrm{~g}$ & $52.64 \mathrm{~g}$ \\
\hline
\end{tabular}

Fig. 6: Tolerance range of exemplary PTFE specimen. Top: Elasticity modulus $E$ and damping factor $\xi$. Middle: Error estimation of $E$ and $\xi$. Bottom: resulting transfer function [6].
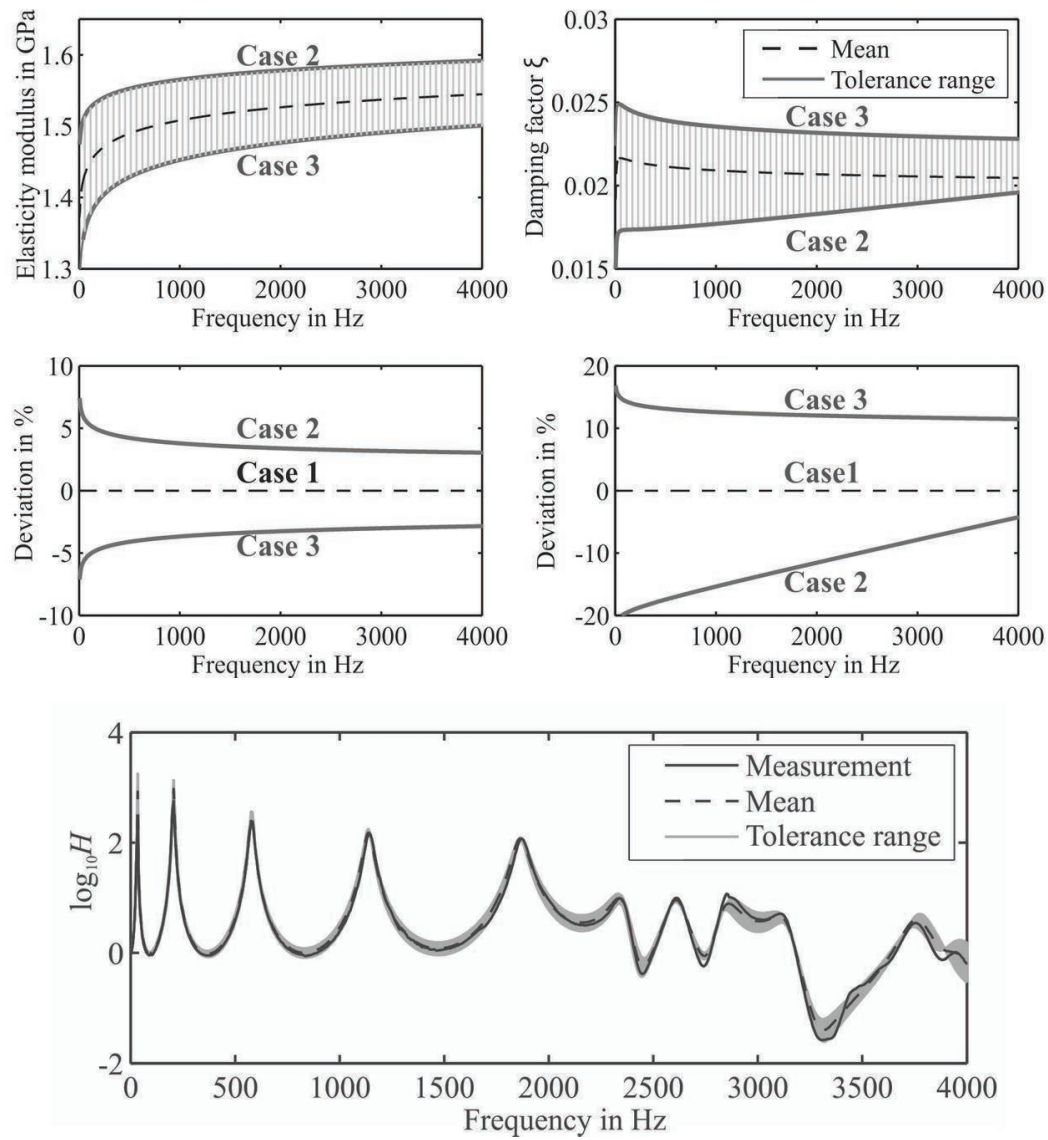


\section{Conclusion and Outlook}

The Inverse Method is a suitable tool for various ill-posed problems in material characterization. The presented approaches enable a precise identification for material parameters of piezoelectric and passive materials. The characterization of piezoceramics features a good repeatability and a precise parameter determination despite a reduction to only two samples contrary to common standardizations. Moreover, the behavior of frequency dependent material parameters can be described by a phenomenological approach. The identified parameters of piezoceramics and passive materials allow precise simulations also for smart composites consisting of a passive material and integrated piezoceramics $[6,8]$.
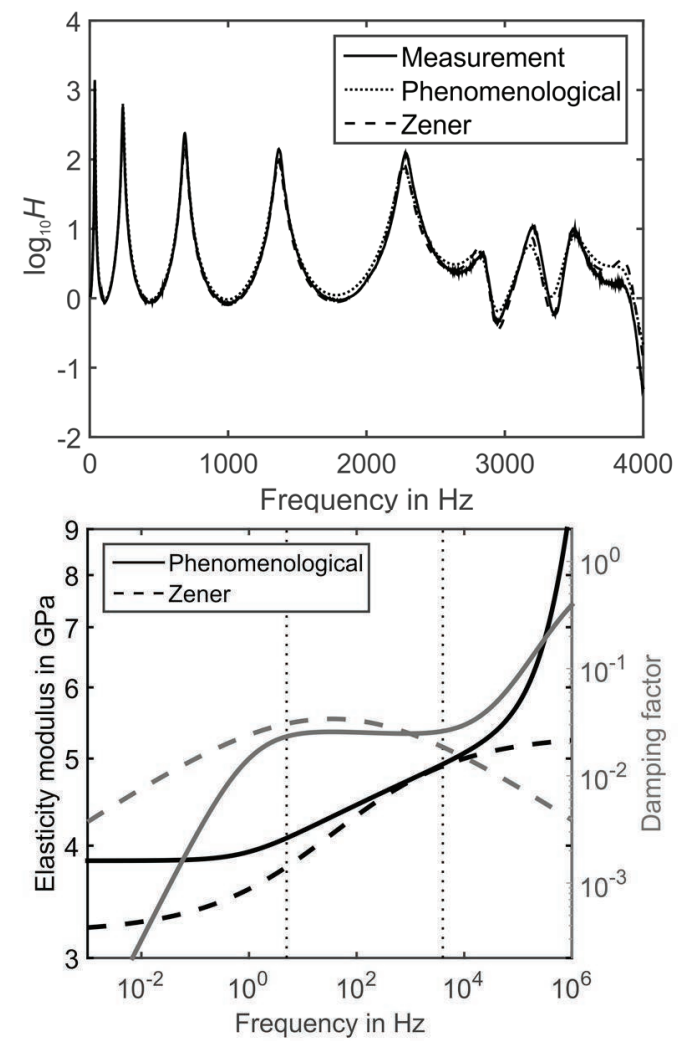

Fig. 7: Comparison between phenomenological approach and Zener model [9] for a PMMA specimen. Top: measurement and simulations of both concepts. Bottom: progression of elasticity modulus and damping factor versus frequency. Vertical dotted lines illustrate frequency range of measurement.

Additionally, new measurement setups for characterization of passive materials are developed to raise the upper frequency limit. The implementation of a fractional derivative model is a basic content of further work to obtain causality in general. Figure 7 shows a comparison between the used phenomenological concept and a four parameter fractional derivative approach based on the Zener model [9].

\section{Acknowledgement}

The underlying research is supported by the Deutsche Forschungsgemeinschaft (DFG) in context of the Collaborative Research Centre/Transregio 39 PT-PIESA, subproject C06.

\section{References}

[1] S. J. Rupitsch, J. Ilg, R. Lerch. Enhancement of the Inverse Method enabling the material parameter identification for piezoceramics, 2011 IEEE International Ultrasonics Symposium, 357360 (2011); doi: 0.1109/ULTSYM.2011.0085

[2] S. J. Rupitsch, A. Sutor, J. Ilg, R. Lerch. Identification Procedure for Real and Imaginary Material Parameters of Piezoceramic Materials, IEEE International Ultrasonics Symposium Proceedings, 1214-1217 (2010); doi: 10.1109/ULTSYM.2010.5935504

[3] IEEE Standard on Piezoelectricity, ANSI/IEEE Standard 176 - 1987; doi: 10.1109/IEEESTD. 1988.79638

[4] J. Ilg, S. J. Rupitsch, R. Lerch. Determination of frequency and temperature dependent mechanical material properties by means of an Inverse Method, In Materials Characterization, Transactions on Engineering Science, 77, 2013. Siena, Italia, 04.-06.06.2013; doi: 10.2495/MC130091

[5] J. Ilg, S. J. Rupitsch, R. Lerch. ImpedanceBased Temperature Sensing With Piezoceramic Devices, IEEE Sensors Journal, Vol. 12, No. 6 (June 2013); doi: 10.1109/JSEN.2013.2256121

[6] J. Ilg. Bestimmung, Verifikation und Anwendung frequenzabhängiger mechanischer Materialkennwerte, Verlag Dr. Hut (2015); ISBN: 978-3-8439-1927-2

[7] S. J. Rupitsch, F. Wolf, A. Sutor, R. Lerch. Reliable modeling of piezoceramic materials utilized in sensors and Actuators, Acta Mech 223, 1809-1821 (2012); doi: 10.1007/s00707-012-0639-7

[8] W. A. Hufenbach, N. Modler, A. Winkler, J. Ilg, S.J. Rupitsch. Fibre-reinforced composite structures based on thermoplastic matrices with embedded piezoceramic modules, Smart Materials and Structures 23 (2014); doi: 10.1088/0964-1726/23/2/025011

[9] T. Pritz. Five-parameter fractional derivative model for polymeric damping materials, Journal of Sound and Vibration 265 (2003); doi: 10.10.16/S0022-460X(02)01530-4

[10] M. O’Donnell, E.T. Jaynes, J.G. Miller. KramersKronig relationship between ultrasonic attenuation and phase Velocity, J. Acoust. Soc. Am. 69(3), March (1981); doi:00014966/81/030696-06S00.8 